\title{
Integral estimation of systemic inflammatory response under sepsis
}

\author{
Natalia V. Zotova ${ }^{1,2}$, Yulia A. Zhuravleva ${ }^{1}$, Tatyana E. Zubova ${ }^{1}$ and Eugeny Y. Gusev ${ }^{1}$ \\ ${ }^{1}$ Institute of Immunology and Physiology of the Ural Branch of the RAS, 106 Pervomayskaya street, Yekaterinburg, Russian \\ Federation \\ 2 The Federal State Autonomous Educational Institution of Higher Professional Education, Ural Federal University, 19 Mira \\ street, Yekaterinburg, Russian Federation
}

\begin{abstract}
Currently, the most significant mediators of the systemic inflammatory response (SIR), specific to the development of critical states in sepsis, have the chaotic changes of concentrations in the blood. The solution to the problem is using integral indicators. A scoring scale of the SIR (0-16 points) is proposed based on the determination in the blood plasma of CRP, TNF- $\alpha$, IL- 6 , IL- 8 and IL-10. The scale was used in the survey of 167 patients with a diagnosis of sepsis (43 patients with sepsis according to definitions of "Sepsis-1 or 2" and 124 patients with sepsis according to the criteria of "Sepsis-3"); septic shock was verified in 31 cases and in 48 cases lethal outcomes were recorded. The association of SIR with critical complications of sepsis was revealed, especially under acute septic shock and in cases of a "second wave" (days 5-7) of critical complications. In contrast, prolonged/ subacute sepsis (more than 14 days) under tertiary peritonitis is characterised by a lesser dependence of the criticality of the state on the severity of SIR. The proposed scale is an open system and allows you to modify the range of used particular indicators that are compatible by pathogenetic and diagnostic significance.
\end{abstract}

Key words: Biomarkers of sepsis - Systemic inflammatory response - Systemic inflammation Integral estimation of inflammation

\section{Introduction}

Sepsis remains a burning social problem up to date. So only in the US, the annual number of all reported deaths from sepsis increased by $31 \%$, from 139,086 in 1999 to 182,242 in 2014 (Epstein et al. 2016).

From a clinical point of view, sepsis is a complex syndrome (an unstable complex of several resuscitation syndromes) caused by the organism's response to infection. A special problem for reducing hospital mortality is numerous cases of latent sepsis. Usually in these cases, the diagnosis of a critical state development and the use of intensive therapy is realised too late. The pathogenesis of sepsis and other critical conditions remains largely an open question and depends on pathology (Chereshnev et al. 2010).

Correspondence to: Natalia Zotova, Institute of Immunology and Physiology of the Ural Branch of the RAS, 106 Pervomayskaya street, 620049 Yekaterinburg, Russian Federation

E-mail: zotovanat@mail.ru
Formally, sepsis is defined as "life-threatening organ dysfunction caused by a dysregulated host response to infection" (Singer et al. 2016). In medical practise, sepsis is a critical infection that requires intensive therapy. This is due to the fact that the last formal criteria of sepsis (a new definition of sepsis (Sepsis-3) was developed in 2016) (Singer et al. 2016) verifies, but does not reveal the pathogenetic nature of multiple organ dysfunction (MOD). The former definitions of sepsis, namely, Sepsis-1 (1991) and Sepsis-2 (2001) reduced this concept to the formula of definitions: "sepsis" = infection + criteria of systemic inflammatory response syndrome (SIRS); "severe sepsis" = sepsis + organ dysfunction and "septic shock" (SS) = sepsis + critical hypotension (not stopped by vasopressors) (Balk 2000). The SIRS criteria are simplified derivatives of scales assessing the general condition of a patient having anything to do with inflammation, namely specific values: tachycardia, tachypnea, leukocytosis or leukopenia and fever (Bone et al. 1992). At the same time, two criteria out of four are sufficient for the verification of SIRS. The low specificity of the SIRS criteria for the development of critical states is obvious (Drewry and 
Hotchkiss 2015; Ter Horst 2016). This problem was solved by the arbitrariness of the verification of SIRS outside the intensive care units. In Sepsis-2, two optional criteria were added: the blood-defined C-reactive protein (CRP) and procalcitonin (PCT), but this did not solve the problem in principle. Currently, PCT, along with presepsin, is mainly used to monitor the effectiveness of antibiotic therapy and to exclude viral sepsis (Vincent and Beumier 2013; Dunne 2015; Mat-Nor et al. 2016). According to the last definition, Sepsis-3 (Singer et al. 2016), sepsis is equal to an infectious, as a rule, bacterial, variant of MOD. It is verified with the sequential (sepsis-related) organ failure assessment (SOFA scale), and under the screening survey, the quick SOFA is applied: tachypnea, alteration in mental status and hypotension. For preliminary verification of sepsis, at least two quick SOFA criteria must be present. The definition "severe sepsis" was abolished. The main criterion of SS verification is vasopressor therapy needed to elevate mean arterial pressure $(\mathrm{MAP}) \geq 65 \mathrm{~mm} \mathrm{Hg}$ and lactate $>2 \mathrm{mmol} / \mathrm{l}(18 \mathrm{mg} /$ dl) despite adequate fluid resuscitation. The new version brought the formal criteria of sepsis closer to the heuristic decisions of doctors. In general, the concept of Sepsis-3 puts an assessment of the condition's criticality in the foreground, and on the back - an assessment of pathogenesis.

Meanwhile, the lack of a holistic image of the pathogenetic core of sepsis invariably leads to "circling" in the solving of particular clinical problems (Askim et al. 2017; Polat 2017). This specifically limits the potential of pathogenetic therapy. One of the directions of problem solving may be the consideration of the pathogenesis of critical states, including infectious, from the position of systemic inflammation (SI) as a common pathological process. This process differs from classical inflammation in a number of following features (Zotova et al. 2016; Gusev and Zotova 2019): 1) SI develops in response to systemic alteration - the effect of trigger inflammatory factors in the bloodstream; 2) the essence of the process is delocalisation of the mechanisms of the inflammation's focus, including the reaction of microvessels; 3) microcirculatory disorders are the main pathogenetic mechanism of clinical manifestations of SI (i.e., shock and MOD); 4) the development of SI is not attributable to the processes of the inflammatory focus, for example, SI can be initiated in an experiment by intravenous administration of microbial antigens to animals; 5) SI is a priori dysfunctional system or process that does not have a protective value for the organism and is also the main cause of deaths in intensive care units.

SI verification requires the specification of the nature of the systemic inflammatory response (SIR). From the above positions, SIR can be considered as a manifestation of generalised (under SI) or local pro-inflammatory cellular stress. Criteria for differentiation of SIR can be certain changes in blood concentrations of inflammatory mediators. However, the key problem is the nonlinearity of these changes and the impossibility to separate clearly the local (with subsequent generalisation in the blood flow) and systemic realising of SIR factors.

The aim of the study is to substantiate some methodological and methodical solutions to the problem of the SIR concretization on the example of sepsis.

\section{Commonly used SIR indicators}

Currently, more than 200 cytokines (mediators of inflammation and immunity of a protein nature) are revealed, and approximately 30 to 40 of them can be considered as real or promising markers of SIR. With that, to be used as SIR marker, cytokine should meet several described below requirements.

It is known, that the family of chemokines includes four subfamilies, approximately 50 cytokines. In clinical practice and experiments, chemokine markers of two subfamilies have been tested: 1) CXCL1 (NAP-3), CXCL2 (MIP-2a) and CXCL8 (IL-8); 2) CCL2 (MCP-1), CCL3 (MIP-1 $\alpha$ ) and CCL5 (RANTES) (Pierrakos and Vincent 2010; Gusev and Chereshnev 2013; Vincent and Beumier 2013; Das 2014). Their strength is a direct connection with endotheliocyte activation and platelet degranulation, a high amplitude of changes in the blood, while the pronounced abnormality of their concentration's distribution in the blood of patients might be considered as the weakness. Potentially, some other chemokines may also be used as indices of SIR: CXCL-7, 9, 10, 11, 17, 20; CCL20 (MIP-3a); and for acute viral infections - CXCL-10 (IP-10).

A special pathogenetic role in the development of SI is played by members of the cytokine families interleukin 1 (IL-1) and tumour necrosis factor (TNF), namely, IL-1 $\beta$ and TNF- $\alpha$. However, the highly pathogenetic role of these cytokines is far from consistent with their prognostic value for assessing the development of critical complications in sepsis (Pierrakos and Vincent 2010). This is due to the feedback mechanisms that limit the jump of these potentially dangerous cytokines in the blood, which are capable (in high concentrations) of pathologically activating microvessel cells (Gusev and Chereshnev 2013). According to our data, IL-1 $\beta$, in comparison with TNF- $\alpha$, is less effective as a criterion of SIR in sepsis and acute trauma because of its low sensitivity and specificity (Gusev et al. 2008). Therefore, we did not widely use IL-1 $\beta$ in our work, despite the fact that the production of IL- $1 \beta$ by different types of cells is a direct objective sign of pro-inflammatory cellular stress - the formation of inflammasomes (Sharma and Kanneganti 2016).

Meanwhile, a number of other cytokines, mainly secreted by macrophages, including stellate endotheliocytes and other types of stromal macrophages of microvessels (e.g., IL-12, IL-18, macrophage migration inhibitory factor (MIF), and 
granulocyte-macrophage-colony stimulating factor (GMCSF) (Pierrakos and Vincent 2010; De Backer et al. 2011; Vincent and Beumier 2013; Sharma and Kanneganti 2016) can be currently used.

One of the most important cytokine markers of SIR in sepsis is IL-6. This factor, secreted in large quantities in SI by vascular macrophages and endotheliocytes (taking into account the total mass of these cells), has a very significant range of changes in blood concentration (up to 1000-fold changes relative to the upper level of the norm and these changes are associated with severity of patients) (Gusev et al. 2008).

The pro-inflammatory cytokine IL-2 is connected with the state of patients with sepsis (De Backer et al. 2011), but has a significantly lower amplitude of its change in the blood. At the same time, some other inflammatory cytokines, for a number of reasons, have not been used yet as criteria for SIR (e.g., IL-17 A and IL-17F are T-helper 17 activation products).

Two anti-inflammatory cytokines, IL-10 and interleukin-1 receptor antagonist (IL-1ra), are described as markers of SIR (Pierrakos and Vincent 2010; Das 2014; Sharma and Kanneganti 2016). They are characterised by a pronounced increase of their levels in the blood under sepsis and by the connection of these changes with the development of critical complications. Perhaps, in SI, systemically activated macrophages of microvessels, rather than leukocytes of the inflammation focus, may be the main source of these cytokines in the blood.

Anti-inflammatory cytokines, IL-4 and IL-13 conditionally, are less useful for this purpose, since their concentrations in sepsis usually do not exceed the upper level of norm by more than two to four times. They are predominantly secreted by activated $\mathrm{T}$ helper type 2 cells, but only in small amounts by stromal cells (including endotheliocytes).

It is reasonable for transforming growth factor beta (TGF- $\beta$ ) to be taken individually. In physiological conditions, this conditional anti-inflammatory cytokine is secreted mainly by stromal macrophages, is deposited in thrombocytes and shows abnormally high levels in the blood. Therefore, it is attributed sooner to anti-inflammatory factors than SIR factors. Thus, according to our data, a decrease of TGF- $\beta$ concentration in the blood relative to the low value of the norm by more than two times is an unfavourable prognostic sign in acute sepsis (Mat-Nor 2016; Zotova et al. 2016).

As for markers of SIR in bacterial sepsis, different kinds of type 1 interferons can be used (Mahieu and Libert 2007). Most of the cells in the human organism are able to release them. At the same time, the use of interferon gamma (IFN- $\gamma$ ) for this purpose meets with the inconsistency of the literature data.

Among the growth factors secreted by activated endotheliocytes, platelet-derived growth factor (PDGF) (Pierrakos and Vincent 2010) and cytokines of the vascular endothelial growth factor (VEGF) family can be highlighted (Karlsson 2008).

The soluble forms of the cytokine receptors for IL-2 (sIL2R) and the TNF family (soluble fragments of several types of receptors) can be used as criteria for SIR (Pierrakos and Vincent 2010; De Backer et al. 2011; Wu et al. 2011). According to our data, sIL-2R can be used to identify SIR, but rather as an addition to the main criterion (Takala et al. 1999).

Signs of systemic activation of microvessels can be considered the accumulation of cast-off endothelial cells (CD144+ cells) in the bloodstream, as well as the soluble forms of certain adhesion receptors: endocan, E-selectin, VCAM-1 and ICAM-1 (Pierrakos and Vincent 2010; Dunne 2015; Kiral 2016). However, sE-selectin and sICAM-1, according to our data, can only be considered as additional criteria to estimate SIR (i.e., a relatively small amplitude of changes in blood concentration in sepsis and acute trauma) (Zotova et al. 2016).

A number of inducible proteins of heat shock can be regarded as promising markers of SIR (Pockley 2008; Gusev and Chereshnev 2013 ). Primarily, these proteins function intracellularly as chaperones, but a certain part of them can be secreted as factors of intercellular communication. These cellular stress factors can modulate cell activity by binding to external and intracellular pattern-recognition receptors (PRRs) (Gusev and Chereshnev 2013).

Soluble fragments of some PRRs are markers of sepsis, namely, presepsin (CD14-co-receptor fragment of Toll-like receptor 4 (TLR-4)) and soluble triggering receptor expressed on myeloid cells-1 (sTREM-1) (another co-receptor of TLR-4) (Vincent and Beumier 2013). TLR-4 in different cell types and platelets is the main receptor that binds LPS gram-negative bacteria. As well as the soluble forms of some scavenger receptors, e.g. sCD163 (De Paoli et al. 2014) and sCD206 (Sertaridou et al. 2015) can also be effectively used as markers of sepsis.

Also among the non-cytokine protein factors of SIR, the non-histone chromosomal protein HMGB1 can be identified. Its level in the blood correlates with the criticality of the patient's condition under sepsis (Huang et al. 2010; Pierrakos and Vincent 2010). However, HMGB1 cannot be unambiguously considered only as a factor of SIR, as it is secreted not only by activated monocytes and macrophages but also enters the bloodstream as a decay product of various tissues, consequently, as a result of necrosis and pyroptosis. This cytokine-like factor acts on the cells via PRRs (i.e., receptor for advanced glycation endproducts (RAGE), TLR-2, 4, 9) (Gusev and Chereshnev 2013).

Proadrenomedullin (pro-ADM) is characterized to reveal a cytokine-like behavior in sepsis, and its concentration in the blood has a good prognostic value (Valenzuela-Sánchez et al. 2016). Thus, pro-ADM is considered one of the popular biomarkers with varying physiological functions, including 
vasodilatory, anti-inflammatory and antimicrobial activity (Andaluz-Ojeda et al. 2017).

Procalcitonin can be secreted into the blood by various stromal cells, but its pathogenetic role is unclear. It, like presepsin, is relatively specific to a bacterial infection, but it has a longer lag-accumulation period in the blood (MatNor 2016).

Some of the acute phase proteins of the liver have traditionally been applied as markers of SIR (Pierrakos and Vincent 2010; Vincent and Beumier 2013; Dunne 2015). Their strength is the relative stability of the upward trend in the blood in various inflammatory processes and widespread use in various areas of practical medicine, and the weak one is the lack of direct connection with systemic cellular stress, as well as the relatively long lag-accumulation period and half-life from the blood flow. The most popular acute phase protein is CRP. In neonatal sepsis, the LPS-binding protein (LBP) is widely used. However, according to our data, it has no appreciable advantages over CRP for the identification of SIR in sepsis in adult patients.

Neopterin, some lipid mediators (e.g., many eicosanoids) (Das 2014; Dalli et al. 2017) and a platelet-activating factor (Ayala and Chaudra 1996), as well as the factor of vasodilation and permeability of microvessels (NO-radical) and the products of its metabolism can be considered as potential non-protein markers of SIR (Pierrakos and Vincent 2010; Silver 2011).

According to Pierrakos and Vincent (2010), "in view of the complexity of the sepsis response, it is unlikely that a single ideal biomarker will ever be found. A combination of several sepsis biomarkers may be more effective, but this requires further evaluation". We believe that this applies not only to sepsis, but also to the evaluation of SIR.

\section{Materials and Methods}

The object of the study was a blood plasma stabilised by citrate, previously frozen at $-20^{\circ} \mathrm{C}$.

The closed system for immunochemiluminometric assay, Immulite (Siemens Medical Solutions Diagnostics, USA), was used as the instrument base.

\section{Concept development}

In this section, the authors found it necessary discussing the concept in more detail and determine some common requirements for estimation of SIR.

\section{The concretization of the concept of SIR by exclusion}

To achieve the goal of the study, it is necessary to identify all the main elements of SIR. Obviously, signs of MOD and destruction, in principle, are not related to the category of genetically determined responses to systemic alteration. The programmed response of the organism to various injuries is very diverse. In this variety, it is rational, as already noted, to isolate SIR "in a restricted sense" and characterise it as an obvious or probabilistic sign of systemic cellular stress. At the same time, SIR indicators (e.g., mediators of inflammation in the blood) must necessarily reflect the pro-inflammatory response of microvessels, as an essential sign of SI. From that position, it is reasonable to separate a number of other system processes from the SIR "in a restricted sense".

Firstly, one of these system processes is the reaction of the hypothalamic-pituitary-adrenal axis and some other manifestations of classical inflammation, which G. Selye defined in 1936 as a "general adaptation syndrome", which had both of physiological and pathological significance. However, just the changes are reflected by SIRS criteria. It is also not advisable to associate signs of metabolic dysfunction with SIR (e.g., increased levels of glucose and lactate in the blood) (Pockley et al. 2008; Pierrakos and Vincent 2010), because, in most cases, these signs are associated with the stress reaction of the neuroendocrine system or are the molecular manifestations of organ dysfunction.

Secondly, one more process is the pathological intravascular activation of phagocytic leukocytes and the release of cytotoxic factors into the bloodstream, which are mechanisms leading to the development of secondary systemic alteration - the driving force of self-reproduction of SI (Gusev and Zotova 2019). This process does not directly reflect the systemic nature of cellular stress. In this case, indications of activity of intravascular phagocytes in SI are, as a rule, unstable and depend on a complex of heterogeneous processes. These processes include the following: strengthening the processes of apoptosis and programmed necrosis (pyroptosis) in the intravascular medium, multidirectional changes in leukopoiesis, abnormalities of migration processes (in particular, homing) and depositing, hardening of adhesion of mature leukocytes on the endothelium of microvessels and migration of leukocytes to the focus of inflammation. In addition, the number of intravascular phagocytes and lymphocytes is insignificant relative to the total mass of endotheliocytes, stromal macrophages and lymphoid tissue in the body. With that, a well-known criterion of immune dysfunction in sepsis is a decrease in number of mature monocytes (HLA-DR+) in the blood (Monneret et al. 2011; $\mathrm{Wu}$ et al. 2011). It also depends on the range of all these processes. Therefore, we do not find it judicious to regard it as a criterion of SIR "in a restricted sense."

To register autophagocytic pathology in sepsis, various products of degranulation of neutrophils and other granulocytes are usually detected in the blood. In particular, according to our data, the cationic protein of eosinophils (ECP) can also be used for this purpose (Gusev et al. 2008). 
Thirdly, microcirculatory disorders, which in sepsis develop mosaically at the level of individual microcirculatory units, at present, can be studied in vivo (e.g., using the method of sidestream dark field microscopy) (De Backer et al. 2011; Bezemer et al. 2012). This phenomenon has a number of features that go beyond the scope of SIR "in a restricted sense." Therefore, it is reasonable to characterise it as an independent functional block of SI. A separate component of this phenomenon is the process of microthrombogenesis, which is fixed in the clinic using criteria of the disseminated intravascular coagulation (DIC) syndrome (Wada et al. 2014; Costello and Nehring 2019). Microcirculation disorders, systemic activation of endotheliocytes and haemostasis factors are closely interrelated with intravascular activation of the kallikrein-kinin and complement systems (Bossi et al. 2011). In this respect, the increase in the blood of anaphylaxins of complement (C3a, C5a) and kinin and the depletion of plasma kallikreinogen (characteristic of critical states in sepsis) can also be attributed to the phenomenon of microcirculatory disorders (Ricklin et al. 2016).

Fourthly, the overcoming of the functional barriers of anti-inflammatory resistance by factors of systemic alteration is a necessary process for SI development. These barriers include antioxidant and antiproteinase systems of blood plasma, as well as the function of "scavengers" of blood flow, realised by a number of protein factors of blood plasma and macrophages of microvessels of the liver and some other organs. Among factors of systemic anti-inflammatory resistance are also several acute-phase proteins: $\alpha 1$-proteinase inhibitor ( $\alpha 1$-antitrypsin), a1-antichymotrypsin, haptoglobin, a1-acid glycoprotein, ceruloplasmin and some others. These proteins of the blood plasma can characterise SIR to a certain extent. However, not an increase in concentrations of these acute phase proteins in the blood, but on the contrary, the depletion of their barrier function in the course of development of systemic damage is advisable to be considered as a phenomenon of SI. Among the negative acute-phase proteins (which are usually reduced in the blood in sepsis), albumin is especially prominent. The assessment of not only the concentration but also the ligand-binding capacity of albumin may be important (Gusev and Zotova 2019). As a rule, depletion of fibronectin and gamma globulin in the blood also has negative value in sepsis. Decreases in a number of factors of blood coagulation, as already noted, characterises the phenomenon of systemic microthrombogenesis in post-capillary venules (Gusev and Zotova 2019), which is clinically referred to as DIC syndrome. On this point, rating thrombocytopenia to signs of MOD (in particular, the SOFA scale) gives rise to certain doubts. This makes the SOFA scale eclectic, aimed to fix not only MOD but also to a certain degree register the patient's general condition.

Fifthly, it is rational not to associate the changes of series of sepsis markers that reflect the organ specificity of the process (e.g., the acute-phase protein neutrophil gelatinaseassociated lipocalin (NGAL), an early marker of acute renal damage) (Panich et al. 2017), with the SIR "in a restricted sense". It is also not suitable to consider an increase in plasma histohormones as SIR, as histohormones are functionally not directly associated with pro-inflammatory cellular stress (e.g., angiopoietins (Ang1, Ang2) and adrenomedullin of endotheliocytes (Pierrakos and Vincent 2010; Dunne 2015) or the fat cell histohormone leptin (Maruna et al. 2001)). Markers of activation and degranulation of mast cells (e.g., blood plasma tryptase) can characterise only the particular manifestations of SIR, but not a holistic image of this phenomenon.

Detection of inducible gene expression by mRNA identification in cells in the experiment allows differentiating the manifestations of cellular stress in different organs, but not at the level of the phenotype of cells and the organism as a whole. Histochemical examination of the cell's phenotype allows determining the degree of mosaicism of this process, but only at the level of an individual organ or tissue. In addition, when using these methods, it is not always possible to distinguish accurately the lifetime changes from postmortem changes.

\section{Some common requirements for estimation of SIR in the "restricted sense"}

A response of the organism to an alteration, including the cytokine network formation (Ter Horst et al. 2016), can be considered as the dynamic nonlinear system (Higgins 2002). A key feature of nonlinear systems is that the relationship between cause and effect is not proportional and the components of a nonlinear network interact to produce unpredictable effects (Rioux 2012). Though nonlinear systems may behave unpredictably, they nevertheless appear to exhibit a universal set of responsive patterns, such as abrupt nonlinear transitions (Rioux 2012). Precisely such abrupt transitions are markers of qualitative changes in the response of the organism to damage. In the context of this article, the authors talk about qualitatively different levels of response, which can be determined by the concentration of inflammatory mediators in the blood.

The nonlinearity of the change in the concentration of blood serum indices in sepsis predetermines the need to use an integral criterion of SIR combining at least three to five individual indicators on an alternative basis (Zotova et al. 2016). These indicators must meet the following requirements:

1. Indicator have to be associated with systemic activation of microvessel cells, platelets and pro-inflammatory systems of blood plasma (i.e., haemostasis, complement and kallikrein-kinin systems) and with clinical manifestations of microcirculatory disorders, primarily, the shock 
states. In other words, it must reflect some probability of development of other key phenomena of SI. At the same time, the individual pathogenetic factors can also serve as indicators of several phenomena depending on specific patterns of their use.

2. Indicator must have a well-known biological and clinical significance.

3. Indicator must have a sufficiently high amplitude of quantitative changes in the blood in pathology, as well as clearly established reference intervals of their concentrations in norm.

4. Indicator has to be distinguished by a relatively short half-life from the bloodstream (to assess the dynamics of the process).

5. The empirical indicators chosen for the integral criterion should reflect, in varying degrees, the SIR image integrity (i.e., include factors with different functional characteristics such as mediators not only with activation but also with immunosuppressive targeting).

6. Due to high amplitude of quantitative changes in the blood, for each indicator several value ranges of their concentrations should be determined. These ranges should be comparable in pathogenetic significance and reflect the probability of development of systemic cellular stress.

Otherwise, comparable value ranges of different indicators should verify specific levels of SIR in the same manner, and these levels of SIR should reflect the varying degree of probability of SI development. In this case, there is not a need for only formal logic to be used to determine SIR levels: "yes" is a definite truth and "no" is its absence. It is also calls for rational multiple probabilistic logic (Bergmann 2008) to be applied as well, for example, in the following sequence of variants of SI signs: 1) "no"; 2) "rather no"; 3) neither "no" nor "yes" (zone of uncertainty); 4) "rather yes" and 5) "yes."

\section{Pathogenetic ranges of selected markers}

At the preliminary stage of the study, five indicators of SIR were selected (to estimate SIR "in a restricted sense"): CRP, TNF- $\alpha$, IL-6, IL-8 and IL-10 (Gusev et al. 2008). For each factor, the upper meaning of the norm (UMN) was detected in plasma of 50 conditionally healthy people: CRP, $10 \mathrm{mg} / \mathrm{l}$; TNF- $\alpha, 8$ pg/ml; IL- 6, 5 pg/ml; IL-8, 10 pg/ml, IL-10, 5 pg/ $\mathrm{ml}$. The rate of excess UMN for each indicator was not more than one case (2\%). Further, for each factor, the serial numbers of ranges of concentrations in plasma were established according to their multiplicity of UMN-exceeding. These ranges were labelled (figured) numerically as different levels meaning different pathogenetic and diagnostic significance, which are the following: 0 - standard level; 1 - level of SIR excluding the development of acute SI (characterises only local products in the focus of inflammation); 2 - typical for SIR under classical inflammation in expressed purulent process as well as under hypoergic (depressive) variants of SI development; 3 - area of ambiguity (does not allow to differentiate classical and systemic inflammation); 4 - typical for hyperergic SI variants (phlogogenic stroke phase), unlikely under classical inflammation; 5 - confirms the development of SI and characterises the criticality of the patient's condition regardless of both SOFA and general condition assessment scale values. In this case, ranges of plasma concentrations of TNF- $a$, IL- 6 and IL- 8 were divided into all six serial level numbers (0-5); CRP only into four levels (0-3), since classical acute-phase proteins do not directly associate with the systemic activation of microvessels; and IL-10 received an additional value (6), which reflects a high probability of death - the stage of SI irreversibility. The ranges for each factor were calculated singly based on the analysis of both our own data and other databases and their generalisations (Gusev et al. 2008).

\section{Calculation of the integral index - coefficient of reactivity scale (CR)}

The sum of the three highest levels (based on ranges of pathogenetic significance mentioned above) of 5 factors used (2 of the 5 smallest levels were excluded) determines the value of the CR in each case. This allowed us to adapt the integral system to the individual features of SIR in each patient. Thus, the range of CR scale is a score from 0 (normal) to 16 points. Comparatively, a CR scale score $\geq 14$ points can accurately verify the SI process, a score of $12-13$ points can detect SI development with a high probability, while $0-4$ points excludes acute SI. A range of 5 to 11 points forms a transition zone (uncertainty). For its concretization, it is necessary to use criteria that reflect other phenomena of SI. Therefore, the CR scale is included as a component of a more fundamental scale of SI. The application of the SI scale, including under sepsis, was presented in our previous publication (Zotova et al. 2016).

\section{Patients}

The following groups were studied:

N1. Deep shin phlegmon - Level III-IV of soft tissue damage in military men; in all patients, signs of SIRS and MOD were shown (average score on a scale of SOFA (Mean $\pm \sigma)=3.20 \pm 1.14$, with the maximum possible value of the scale being 24 points). The group formally corresponds to the definition of "sepsis" according to consensus "Sepsis-3" (Singer et al. 2016). The dominant aetiological factor was Staphylococcus aureus. The study was conducted immediately after the surgical treatment of the inflammatory focus. Deaths and shock states in the postoperative period were 
not observed; treatment was carried out only in the surgical department $(n=40$, age (Mean $\pm \sigma)=19.0 \pm 0.9$ years $)$.

N2. Sepsis (bacterial, "Sepsis-1" according to consensus "Sepsis-1" (Bone et al. 1992)), 1-2 days of hospitalisation. Initial diseases: severe pneumonia, peritonitis, obstetrical sepsis and some other reasons. Some patients had dysfunction of one system only (severe sepsis), but no signs of MOD $($ SOFA $($ Mean $\pm \sigma)=1.13 \pm 0.81, n=31$, mean age $=41.1 \pm$ 18.0 years), and all patients in this and in other groups went through intensive therapy in the intensive care unit (ICU).

N3. Severe sepsis with MOD ("Sepsis-3") (SOFA (Mean $\pm \sigma)=5.50 \pm 2.30, n=46$, mean age $=50.1 \pm 16.6$ years $)$ and 1-2 days from hospitalisation in the ICU.

N4. The same in addition to septic shock under acute process, SS-AP (the presence of hypotension not responding to vasopressors) (SOFA (Mean $\pm \sigma)=9.75 \pm 2.22, n=14$, mean age $=54.9 \pm 16.4$ years $)$.

N5. Sepsis without MOD ("Sepsis-1") (SOFA (Mean $\pm \sigma)$ $=0.75 \pm 0.87$, screening at day $5-7$ of hospitalisation in the ICU, $n=12$, mean age $=40.2 \pm 15.7$ years).

N6. The same, but with MOD ("Sepsis-3") (SOFA (Mean $\pm \sigma)=5.77 \pm 2.20, n=13$, mean age $=37.7 \pm 15.4$ years $)$.

N7. Tertiary peritonitis with MOD, and prolonged subacute septic process - more than 14 days from the date of hospitalisation in the ICU (SOFA (Mean $\pm \sigma)=4.55 \pm 2.72$, $n=34$, mean age $=51.5 \pm 16.6$ years). These variant septic processes can be considered as transition zones between acute and subacute sepsis.

N8. The same in addition to development of septic shock, SS-SAP (SOFA (Mean $\pm \sigma)=8.06 \pm 3.23, n=17$, mean age $=50.2 \pm 15.6$ years $)$.

N9. Control group, practically healthy people - blood donors (aged 18-55 years) and elderly people (aged 60-83 years) without acute and system destructive diseases, as well as without acute attack of chronic diseases $(n=72$, mean age $=44.64 \pm 18.41$ years $)$.

The research was approved by Institutional Ethics Committee and informed written consent was obtained.

N10. Lethal outcomes - integral group included all fatal cases of groups N3, N4, N6, N7, N8, $(n=48$, mean age $=$ $48.46 \pm 15.68$ years $)$.

\section{Statistical analysis}

Statistical analyses were performed using SPSS for Windows 15.0 (SPSS Inc., Chicago, IL, USA), MedCalc version 7.4.4.1 for Windows (MedCalc Software) and Statistica 6.0 programme (Stat Soft, Inc., USA). The descriptive statistics are presented by their main characteristics: $\mathrm{m}-$ mean value, $\mathrm{Me}-$ median, $\sigma-$ standard deviation, and $25 \% \div 75 \%$ - quartiles. Testing of the hypothesis that the distribution of samples was not normal was made by KolmogorovSmirnov and Shapiro-Wilk tests. Non-parametric analytical methods were used: Spearman correlation coefficient (r), comparison of two independent groups by Mann-Whitney $\mathrm{U}$ test, comparison of several independent groups using the Kruskal-Wallis $\mathrm{H}$-test and a median test with a minimum level of statistical significance of $p<0.05$. Parametric analytical methods (Newman-Keuls and Duncan) were also used to compare more than two independent samples. Comparisons between groups were performed using the Chi-square $\left(\chi^{2}\right)$ test for categorical variables. Areas under receiver operating characteristic curve (AUC) were used to evaluate the ability of IL-6, IL-8, IL-10, TNF- $\alpha$, CRP, CR and SOFA score to discriminate survivors from non-survivors, as well as patients with SS-AP from patients without SS-AP. AUC were pairwise compared. Statistical significance was defined as a $p$-value $<0.05$.

\section{Results}

In most cases, the empirical data on cytokines in control (conditionally healthy) patients were below the method's sensitivity ( $2 \mathrm{pg} / \mathrm{ml}$ for IL-6, $5 \mathrm{pg} / \mathrm{ml}$ for IL- 8 and IL- 10 and $4 \mathrm{pg} / \mathrm{ml}$ for TNF- $\mathrm{a}$ ). In those cases, the values of the indicators were equated with the threshold of test-sensitivity. Only four of the 72 people in the control group (all over the age of 60$)$ had tempered signs of SIR ( $C R=2-3$ points). On the contrary, all patients of the groups with the criteria Sepsis-1 and Sepsis-3 had signs of SIR (CR $\geq 2$ points). Conditionally healthy people showed all factors of SIR differed from septic patients ( $p<0.05$, by Mann-Whitney criterion), except IL-10 in group N1. In view of the obvious differences in the control group with all groups of septic patients, this group was subsequently excluded from the statistical analysis.

\section{Testing of the distributions of SIR indicators in groups}

The distribution of integral coefficient CR (Table 1) conformed to a normal distribution in all eight investigated sepsis groups tested by the Kolmogorov-Smirnov test $(0.20$ $<p<0.10)$ and Shapiro-Wilk test $(p>0.17)$ in four groups (groups N1, 4, 7 and 8). Accordingly, the normal distribution of CRP was noted in six groups (group 2 and 4-8) according to the Kolmogorov-Smirnov test, but according to the Shapiro-Wilk test, there was one case exhibiting normality of CRP distribution in group N4. The distribution of cytokines corresponded to normal only in some cases according to the Kolmogorov-Smirnov test (IL- 6 - group N2, TNF- $\alpha$ - group N8, IL-10 - group N4).

Thus, the CR, in contrast to other indicators, is characterised by a relatively normal distribution in most cases. Distribution of CRP conformed to normal only in individual groups, and the distribution of cytokines displayed an abnormal character. 


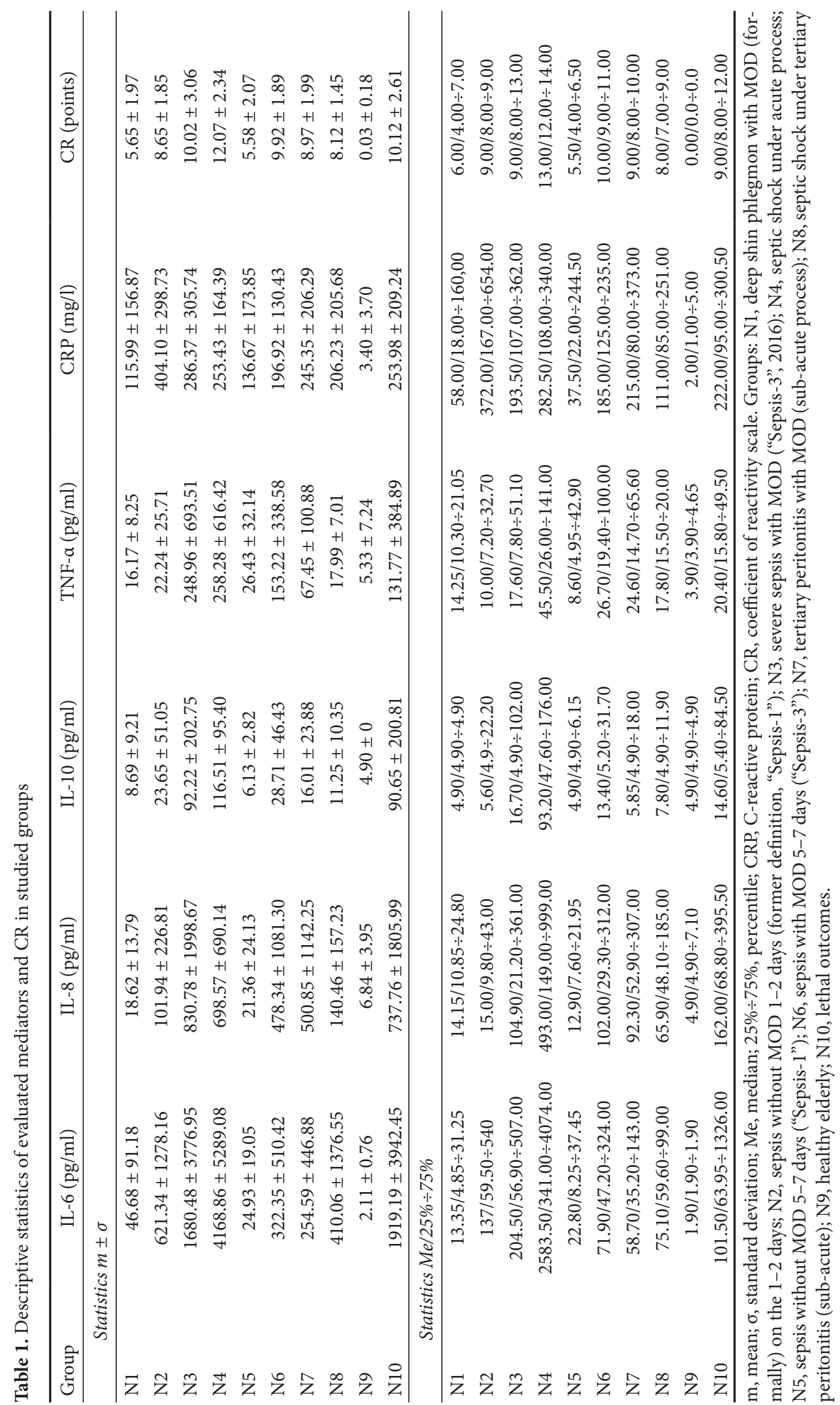


Table 2. Significant differences between the groups for the studied indicators

\begin{tabular}{lcccccc}
\hline Group & CR & CRP & TNF- & IL-6 & IL-8 & IL-10 \\
\hline N1 & $2,3,4,6,7,8$ & $2,3^{*}$ & No & 4 & $3^{*}$ & $3^{*}, 4$ \\
N2 & $1,4,5$ & $1,5,7^{\star}, 8^{\star}$ & No & 4 & No & $3^{\star}, 4$ \\
N3 & $1,4,5,8^{\star}$ & 1 & No & 4 & $1^{\star}, 5^{\star}$ & $1^{\star}, 2^{\star}, 5^{\star}, 7^{\star}, 8^{\star}$ \\
N4 & $1,2,3,5,6,7,8$ & No & No & $1,2,3,5,6,7,8$ & No & $1,2,5,6,7,8$ \\
N5 & $2,3,4,6,7,8$ & 2 & No & 4 & $3^{\star}$ & $3^{\star}, 4$ \\
N6 & $1,4,5,8^{\star}$ & $2^{\star}$ & No & 4 & No & 4 \\
N7 & $1,4,5$ & $2^{\star}$ & No & 4 & No & $3^{\star}, 4$ \\
N8 & $1,3^{\star}, 4,5,6^{\star}$ & $2^{\star}$ & No & 4 & No & $3^{\star}, 4$ \\
\hline
\end{tabular}

Comparison of several independent groups using the Newman-Keuls and Duncan tests parametric analytical methods with a minimum level of statistical significance of $p<0.05 .{ }^{*}$ Significant differences identified by only the Duncan test are indicated. (For abbreviations, see Table 1).

Reduction in the normality of the distribution of CR is only one of the conditions for its use as an integral indicator of SIR. Equally important is its differentiating function in the intergroup analysis.

\section{Multiple intergroup analysis}

\section{Comparison of the indicators}

The best differentiating function of the $\mathrm{CR}$ was revealed with the use of multiple intergroup analysis (Newman-Keuls and Duncan test, $p<0.05)$. So, CR separated the groups into 19 cases out of 28 possible variants comparing all eight groups with each other, IL-10 in 11 cases, IL- 6 in 7, CRP in 5 cases, IL- 8 in 2 cases and TNF- $\alpha$ in no cases (Table 2).

At the same time, the group of patients with SS-AP (obvious clinical manifestations of SI) differed in CR and IL-6 from all seven other groups (in IL-10 from six groups and TNF- $\alpha$, IL-8, CRP did not divide at all from other groups) (Table 3).

Hence, the CR, based on the results of multiple analysis, had the greatest separation function as a criterion of SIR for the studied groups.

\section{Timing of the development of the process on 1-2 days/5-7 days}

In groups without MOD, the tendency of a decrease in the severity of SIR determined by CR, depending on the time of the process development, is typical: CR in group N2 (1-2 days) $>$ CR in group N4 (5-7 days).

However, in patients with MOD, values of CR in group N3 (1-2 days), group N5 (5-7 days) and group N7 (tertiary peritonitis) were not significantly different.

In general, SIR in this study, being the most intense on days 1-2 of the clinical development of sepsis, had decreased significantly by days $5-7$, but remained high in the presence of MOD signs.

The speciality of SIR in group N1, non-resuscitation patients with MOD

This group differed in CR and a number of particular indicators from all resuscitation groups. In this case, it should be noted the effectiveness of the heuristic approaches of physicians with neglect of relatively moderate manifestations of MOD in group N1 (SOFA scores from 2 to 6 points). However, according to our data (Zotova et al. 2016), some patients of group N1

Table 3. Pairwise comparison of groups by Mann-Whitney U test

\begin{tabular}{lclll}
\hline \multirow{2}{*}{$\begin{array}{l}\text { Groups of } \\
\text { patients in critical } \\
\text { states }\end{array}$} & $\begin{array}{c}\text { Lethal } \\
\text { Outcomes }\end{array}$ & \multicolumn{3}{l}{ Groups of non-critical patients } \\
\cline { 5 - 5 } & $(\%)$ & \multicolumn{1}{c}{ N1 } & \multicolumn{1}{c}{ N2 } & \multicolumn{1}{c}{ N5 } \\
\hline N3 & 23.91 & CR, CRP, IL-6, IL-8, IL-10 & CR, IL-8, IL-10 & CR, CRP, IL-6, IL-8, IL-10 \\
N6 & 36.36 & CR, CRP, TNF- $\alpha$, IL-6, IL-8, IL-10 & CR, CRP, TNF- $\alpha$, IL-8 & CR, IL-6, IL-8, IL-10 \\
N7 & 29.41 & CR, CRP, TNF- $\alpha$, IL-6, IL-8, IL-10 & CRP, TNF- $\alpha$, IL-8 & CR, CRP, TNF- $\alpha$, IL-6, IL-8 \\
N8 & 94.12 & CR, CRP, IL-6, IL-8, IL-10 & CRP, IL-8 & CR, CRP, IL-6, IL-8, IL-10 \\
N4 & 71.43 & CR, CRP, TNF- $\alpha$, IL-6, IL-8, IL-10 & CR, TNF- $\alpha$, IL-6, IL-8, IL-10 & CR, TNF- $\alpha$, IL-6, IL-8, IL-10 \\
\hline
\end{tabular}

Parameters by which groups differ in pairwise comparison are indicated. (For abbreviations, see Table 1). 
had borderline manifestations of SI with signs of secondary systemic damage of internal organs. Doctors do not usually fix such changes and their long-term effects are not analysed.

Obviously, in the conditions of practical application of Sepsis-3 criteria, this category of patients, like some patients without MOD (groups N2 and N4 with criteria of Sepsis-1) would be problematic for determining of intensive care indications without using additional criteria for monitoring the patient's condition.

\section{The differentiation of the groups with MOD/without MOD using the Mann-Whitney test}

The differentiation of the groups in terms of criticality had a fundamental importance. Such differences were particularly convincing in the pairwise (with MOD/without MOD) comparison $(p<0.05$, Table 3 ) of groups, which were comparable by period of the development of sepsis:

1) Estimation of SIR in resuscitation patients on days 1-2 of clinical development of sepsis. Group N2 (without MOD) and group N3 (with MOD) differed with regard to CR, IL- 8 and IL- 10 .

2) Assessment of SIR in resuscitation patients on days 5-7 of sepsis development. Group N5 (without MOD) and group N6 (with MOD) varied $(p<0.05)$ in CR, IL-6, IL-8 and IL-10.

Accordingly to the data, in both cases, groups with MOD/ without MOD differed only in CR, IL-8 and IL-10, while the CRP did not show separating function in both cases.

\section{The differentiation of the groups of patients with septic shock under acute/subacute process (SS-AP/SS-SAP) by SIR criteria}

Two groups of SS were separated in multiple intergroup analysis (Table 2) by the values of CR, IL-6 and IL-10. Dif- ferences in the non-parametric Mann-Whitney criterion for groups with SS were even more significant (Table 3 ). The groups were significantly $(p<0.05)$ different by all indicators, except CRP (not shown in Table 3 ).

In general, shock states under subacute/prolonged sepsis were characterised by relatively moderate manifestations of SIR in comparison with shock under acute sepsis.

\section{Correlation between SIR indicators and SOFA scale score in groups}

The data shown in Table 4 are the correlations $(\mathrm{R}>0.4, p<$ 0.05 ) between all indices of the SIR, as well as the SOFA scale scores in all groups (SS is the integral group $(\mathrm{N} 4+\mathrm{N} 8)$ ). In general, a relatively low level of correlations (especially strong $(\mathrm{R}>0.8)$ ) was typical. Therefore, the total number of low/ strong correlations $(\mathrm{R}>0.4 / \mathrm{R}>0.8)$ in the groups was the following: N1 - 11/0, N2 - 12/0, N3 - 28/2, N5 - 3/1, N6 $8 / 0$, N7 - $14 / 2$ and SS - 20/6. In whole, as the state severity increases, the number and the level of correlations between groups increased.

For individual indicators, the number of low/strong correlations $(\mathrm{R}>0.4 / \mathrm{R}>0.8)$ was the following: $\mathrm{CR}-27 / 5$, IL-6 $-21 / 2$, IL-8 - 18/3, IL-10 - 12/1, CRP - 11/0 and SOFA - 7/1. Thus, the SOFA scale score was weakly correlated with the SIR indicators, since they reflected different pathogenetic parts of SI. CR was most closely associated with other indicators of SIR, which confirmed its integrating role.

\section{Estimation of alternative occurrence of particular indicators (3 out of 5) of SIR to the structure of integral criterion CR}

The average probability of inclusion of each of the five particular SIR markers in the CR structure (with the same diagnostic efficiency) was $60 \%$, and the potential probability

Table 4. Correlations of indicators in studied groups

\begin{tabular}{|c|c|c|c|c|c|c|}
\hline Group & $\mathrm{CR}$ & CRP & TNF- $\alpha$ & IL-6 & IL-8 & IL-10 \\
\hline N1 & CRP, IL-6, SOFA, IL-10 & CR, SOFA, IL-6 & No & CR, CRP, SOFA & No & $\mathrm{CR}$ \\
\hline N2 & $\begin{array}{l}\text { TNF- } \alpha, \text { IL-6, IL-8, } \\
\text { IL-10, SOFA }\end{array}$ & No & CR & CR, IL-8, SOFA & CR, IL-6 & $\mathrm{CR}$ \\
\hline N3 & $\begin{array}{l}\text { CRP, TNF- } \alpha, \text { IL-6*, } \\
\text { IL-8, IL-10, SOFA }\end{array}$ & $\begin{array}{l}\text { CR, TNF- } \alpha, \text { IL-6, } \\
\text { IL-8 }\end{array}$ & $\begin{array}{l}\text { CR, CRP, IL-6, } \\
\text { IL-8 }\end{array}$ & $\begin{array}{l}\text { CR }^{\star}, \text { CRP, TNF- } \alpha, \text { IL- } 8, \\
\text { IL-10 }\end{array}$ & $\begin{array}{c}\text { CR, CRP, IL-6, } \\
\text { IL-10, TNF- } \alpha, \text { SOFA }\end{array}$ & $\begin{array}{l}\text { CR, IL-6, } \\
\text { IL-8 }\end{array}$ \\
\hline N5 & CRP & $\mathrm{CR}$ & No & No & SOFA $^{*}$ & No \\
\hline N6 & CRP, IL-6 & CR, TNF- $\alpha$ & CRP & CR, IL-8 & IL-6 & No \\
\hline N7 & $\begin{array}{c}\text { TNF- } \alpha, \text { IL- } 6, \text { IL- } 8^{\star}, \\
\text { IL-10 }\end{array}$ & No & $\mathrm{CR}$ & CR, IL-8, IL-10 & $\mathrm{CR}^{\star}$, IL6, IL-10 & $\begin{array}{l}\text { CR, IL-6, } \\
\text { IL-8 }\end{array}$ \\
\hline $\mathrm{N} 4+\mathrm{N} 8$ & $\begin{array}{l}\text { TNF- } \alpha, \text { IL- } 6^{\star}, \text { IL- } 8^{\star} \\
\text { IL- } 10^{*}\end{array}$ & No & $\begin{array}{l}\text { CR, IL-6, IL-8, } \\
\text { IL-10 }\end{array}$ & $\mathrm{CR}^{*}, \mathrm{TNF}-\alpha, \mathrm{IL}-8, \mathrm{IL}-10$ & $\begin{array}{l}\text { CR }^{\star}, \text { TNF- } \alpha, \text { IL- } 6, \\
\text { IL-10 }\end{array}$ & $\begin{array}{l}\text { CR }^{\star}, \text { TNF- } \alpha, \\
\text { IL-6, IL- } 8\end{array}$ \\
\hline N9 & IL-8 & IL-6 & No & CRP & $\mathrm{CR}$ & No \\
\hline
\end{tabular}

The table shows the values of the Spearman rank-order correlations $(\mathrm{R}>0.04$, low $), p<0.05 .{ }^{*}$ Correlations of indicators with $\mathrm{R}>0.80$ (strong). (For abbreviations, see Table 1). 
was from $0 \%$ to $100 \%$. Actually, in all studied groups, all individual indicators of SIR participated in the formation of the CR with different frequency (Table 5).

The frequency of inclusion of IL- 6 in the CR calculation significantly $\left(\chi^{2}, p<0.05\right)$ exceeded the average level of inclusion of other mediators. CRP was included in CR formation only in non-critical patients.

The frequency of entry of both IL- 8 and IL-10 into the CR calculation was approximately equal to the average level, and the frequency of TNF- $\alpha$ was significantly lower, especially in the groups with SS and lethal outcomes ( 25\%).

Therefore, all factors were involved in the CR formation with varying frequency (in different patients in different combinations).

\section{ROC analysis of SIR indicators and SOFA scale to predict septic shock development and lethal outcomes in groups of patient with acute variant of sepsis}

\section{Development of septic shock}

AUCs for studied indicators and scales were the following $(p<0.05): \mathrm{AUC}_{\mathrm{IL}-8}=0.858 \pm 0.35 ; \mathrm{AUC}_{\mathrm{CR}}=0.834 \pm 0.54$; $\mathrm{AUC}_{\mathrm{IL}-10}=0.834 \pm 0.55 ; \mathrm{AUC}_{\mathrm{IL}-6}=0.829 \pm 0.60 ; \mathrm{AUC}_{\mathrm{SOFA}}$ $=0.828 \pm 0.87 ; \mathrm{AUC}_{\mathrm{TNF}-\alpha}=0.768 \pm 0.57 ; \mathrm{AUC}_{\mathrm{CRP}}=0.588$ \pm 0.73 .

\section{Lethal outcome}

AUCs for studied indicators and scales were the following $(p<0.05): \mathrm{AUC}_{\mathrm{SOFA}}=0.887 \pm 0.51 ; \mathrm{AUC}_{\mathrm{IL}-8}=0.865 \pm 0.29$; $\mathrm{AUC}_{\mathrm{CR}}=0.835 \pm 0.40 ; \mathrm{AUC}_{\mathrm{IL}-10}=0.818 \pm 0.47 ; \mathrm{AUC}_{\mathrm{IL}-6}$ $=0.811 \pm 0.43 ; \mathrm{AUC}_{\mathrm{TNF}-\alpha}=0.662 \pm 0.59 ; \mathrm{AUC}_{\mathrm{CRP}}=0.642$ \pm 0.54 .

Thus, SOFA, CR, IL-8, IL-6 and IL-10 had a "very good" (AUC range is $0.8-0.9$ ) combination of sensitivity and specificity according to the receiver operating characteristic (ROC) analysis criterion in both cases (Zweig and Campbell 1993).

\section{Discussion}

Since the PIRO (predisposition, infection, body response and organ dysfunction) concept has been suggested (Granja and Povoa 2015), the selection of individual and integral risk factors for sepsis development remains an actual problem. At the same time, it is necessary to understand why, with a huge expenditure of resources, the Aesopian language can define the ultimate result of efforts within the framework of this concept as "the mountain spawned a mouse". Probably, there are common problems without the solution of which it is impossible to solve the particular problems, including
Table 5. Frequency (\%) of occurrence of indicators in the coefficient of reactivity $(\mathrm{CR})$ calculation

\begin{tabular}{llllll}
\hline Group & IL-6 & IL-8 & IL-10 & TNF- $\alpha$ & CRP \\
\hline N1 & 69.17 & 51.68 & $20.00^{*}$ & 67.50 & $91.65^{\star}$ \\
N2 & $99.19^{\star}$ & 36.32 & 49.12 & $29.83^{\star}$ & $85.52^{\star}$ \\
N3 & $96.27^{\star}$ & 67.75 & 58.96 & $27.65^{\star}$ & 49.36 \\
N4 & 91.64 & 72.64 & 89.29 & 26.14 & $20.21^{\star}$ \\
N5 & $95.25^{\star}$ & 33.92 & 30.00 & 45.00 & $95.83^{\star}$ \\
N6 & 88.46 & 62.85 & 53.85 & 37.15 & 57.69 \\
N7 & $84.97^{\star}$ & 72.06 & 38.23 & 38.41 & 66.32 \\
N8 & $92.15^{\star}$ & 68.17 & 52.16 & $24.23^{\star}$ & 63.29 \\
N4+N8 & $91.87^{\star}$ & 70.16 & 68.87 & $25.10^{\star}$ & 43.97 \\
N10 & $92.77^{\star}$ & 66.10 & 65.88 & $25.69^{*}$ & 49.54 \\
\hline
\end{tabular}

Average expected frequency of occurrence of the indicator in the $\mathrm{CR}$ calculation is $60 \%$. The table shows the observed frequency of each indicator. ${ }^{*}$ Significant differences of expected/observed frequencies. (For abbreviations, see Table 1).

the realisation of the principle of individual approaches to patients. The modern capabilities of network information systems and medical instrumentation allow us, in principle, to solve these problems more effectively. One of the conditions for this can be new theoretical and methodological approaches for understanding the pathogenesis of sepsis at the level of a whole organism.

Clinical models (nosological and syndromic) are based on the theoretical foundation of common pathological processes. An attempt to reflect the principal image of the pathogenesis of critical states on the basis of the SIRS concept "in wide extent" did not solve this problem. The existing classical ideas about inflammation also do not solve this problem, despite the fact that in the pathogenesis of critical states, pro-inflammatory mechanisms play the crucial role. Thereupon, it became necessary to characterise SI as an independent common pathological process, with a number of attributes that differ from classical inflammation.

Systemic inflammation is a complex of processes, and SIR is only one of its phenomena (functional block). This determines that the principle image of the pathogenesis of sepsis should correspond to a combination of these blocks in different compositions. SIR among the range of these phenomena is the most difficult to assess and formalise. At the same time, SIR itself is being widely interpreted at present and needs to be concretized. It can be defined in a "restricted sense", like the accumulation of markers of pro-inflammatory cellular stress in the bloodstream. The specificity of SIR to SI is not absolute, since the signs of SIR are more or less characteristic in classical inflammation. This requires the ranking of concentration diapasons of each SIR mediator in the blood based on its probabilistic connection with the processes of pro-inflammatory cellular stress of the local or 
organismic level. Changes in the factors of SIR in the blood are chaotic and unstable. Therefore, there is a need to use several indicators formulated as an integral criterion. In this case, the CR scale (0-16 points) was used as the integral index, and TNF- $\alpha$, IL-6, IL-8, IL-10 and CRP became its empirical measures.

The presented data show the association of SIR manifestations typical for SI with critical complications of sepsis, including lethal outcomes and SS. Thus, the majority of patients with SS in acute sepsis have relatively high SIR values ( $C R \geq 11$ points), as in the case of the "second wave" (5-7 days) of critical sepsis complications in the form of MOD with a high probability of death (in group N6: CR $\geq 11$ points in $46.15 \%$ of cases). While, with a more favourable course of sepsis (group N5), the intensity of SIR decreases significantly by $5-7$ days ( $C R \geq 11$ points is fixed in no one case). This pattern of development of acute sepsis determines the possibility of using SIR criteria to predict the development of critical complications. This is confirmed by the ROC analysis of the studied parameters for development of SS (under acute process) and lethal outcomes.

In contrast to acute sepsis, prolonged/subacute sepsis (more than 14/30 days in ICUs) is characterised by a lesser dependence of the state severity on the intensity of SIR. First, this covers the SS group and the severity of SIR in most patients of this group can be referred to as in the "grey zone" (CR 5-10 points). Meanwhile, SI in this category of patients is relatively well verified using a more integrated scale, which takes into account other phenomena of this process (Zotova et al. 2016). In addition, patients with SS-SAP have a vivid picture of the critical state manifested as a complex of resuscitative syndromes with a very high probability of death (94.12\% of cases, group N8). This signs per se is the SI criterion. Various phases of SI development prevail under acute and subacute variants of SS (Zotova et al. 2016).

The integral CR scale applied to estimate SIR can be used as a criterion for the prediction of critical complications due the monitoring of probabilistic latent course of sepsis, for example, in cases close to groups of non-resuscitation patients with MOD (group N1) or resuscitation patients without clear criteria of MOD (groups N2 and N5). As a rule, in clinical practice, the debut of SI development is not diagnosed in time (as there are no universally recognised criteria), but often irreversible phases of SI development with an obvious clinically critical condition are registered.

Among five used indicators of SIR, the following mediators are most often included in the CR structure ( 3 factors out of 5): IL-6 (the most), IL-8 and IL-10. Among them, the minimal rate has TNF- $\alpha$ (but not in all patients). However, TNF- $\alpha$, according to our data, as an indicator of SIR is quite significant in a number of chronic diseases, including systemic autoimmune diseases. This makes it rational to include TNF- $\alpha$ in CR in cases of solving problems wider than registering SIR in sepsis. With regard to sepsis, it is advisable to include presepsin or PCT in the structure of the integral index instead of TNF- $\alpha$ or CRP. At present, the dynamics of changes in the blood concentrations of presepsin and PCT in sepsis have been sufficiently studied for inclusion in the integral index of SIR. However, we did not use these factors extensively, because the aim of our research was to conduct the comparative analysis of SIR in infectious and aseptic processes (Zotova et al. 2016).

The conditions for the clinical approbation of the methodological approaches outlined above are not only the determination of their clinical and economic effectiveness but also the possibility of using such criteria in cooperation with other clinical, laboratory, instrumental criteria, as well as computer software supporting clinical decision making. From this perspective, the proposed version of the integral evaluation of SIR should be considered as one of the possible alternatives to the solution of a particular problem within the framework of the problem outlined above.

Thus, the criteria of SIR estimation "in the restricted sense" and SI as a whole reflect the existence of a common pathogenetic core of critical states (the presence of a common pathogenetic pattern), regardless of their aetiology. In this case, sepsis and especially the development of SS are obvious points of application of these criteria. These criteria do not fully disclose a heterogeneous picture of a specific pathological process (nosological and syndromic). However, without the identification of the pathogenetic core, it is impossible to integrate multiple parameters of specific diseases, including their genetic predisposition, the pathological role of various aetiological, environmental and ontogenetic factors into a single whole.

\section{Conclusions}

- The concept of a SIR in the framework of the pathogenesis of sepsis should be specified as a manifestation of systemic cellular stress.

- At present, there are several dozens of molecular indicators that can be used as SIR criteria.

- Rates of mediators of SIR are characterised by nonlinearity of changes, abnormality of distribution in groups, low degree of correlation with each other and individual features of their correlation in certain patients, which requires integral criteria of SIR to be used.

- SIR can be verified using the integral criterion (CR scale from 0 to 16 points) based on rates of IL-6, IL-8, IL-10, TNF- $\alpha$ and CRP.

- The highest $\mathrm{CR}$ values are characteristic for patients with shock and other critical complications of acute sepsis (especially, under the "second wave" of days 5-7 of the process), while SS in prolonged and subacute sepsis 
(more than 14 days) is characterised by more moderate manifestations of SIR.

- Competitive inclusion in the structure of the integral criterion of 3 of the most pronounced indicators (out of 5) allows us to implement the principle of an individual approach in the evaluation of SIR.

- CR scale is probabilistic in character under SI verification. The scale determines the areas with a very high $(C R \geq 11$ points) or low (CR $\leq 4$ points) probability of SI development, as well as a transition or undefined zone of CR ranging from 5 to 10 points. It requires using criteria of other SI processes for verification of SI development as a whole.

Acknowledgments. The work was carried out within the framework of the IIP UrB RAS theme No AAAA-A18-118020590108-7.

\section{References}

Andaluz-Ojeda D, Nguyen HB, Meunier-Beillard N, Cicuéndez R, Quenot JP, Calvo D, Dargent A, Zarca E, Andrés C, Nogales L, et al. (2017): Superior accuracy of mid-regional proadrenomedullin for mortality prediction in sepsis with varying levels of illness severity. Ann. Intensive Care 7, 15 https://doi.org/10.1186/s13613-017-0238-9

Askim A, Moser F, Gustad LT, Stene H, Gundersen M, Åsvold BO, Dale J, Bjørnsen LP, Damås JK, Solligård E (2017): Poor performance of quick-SOFA (qSOFA) score in predicting severe sepsis and mortality - a prospective study of patients admitted with infection to the emergency department. Scand. J. Trauma Resusc. Emerg. Med. 25, 56 https://doi.org/10.1186/s13049-017-0399-4

Ayala A, Chaudra IH (1996): Platelet activating factor and its role in trauma, shock, and sepsis. New Horiz. 4, 265-275

Balk RA (2000): Severe sepsis and septic shock. Definitions, epidemiology, and clinical manifestations. Crit. Care Clin. 16, 179-192 https://doi.org/10.1016/S0749-0704(05)70106-8

Bergmann M (2008): Fuzzy algebras. In: An Introduction to Many-Valued and Fuzzy Logic: Semantics, Algebras, and Derivation Systems. pp. 212-222, Cambridge University Press, Cambridge https://doi.org/10.1017/CBO9780511801129.013

Bezemer R, Bartels SA, Bakker J, Ince C (2012): Clinical review: Clinical imaging of the sublingual microcirculation in the critically ill--where do we stand? Crit. Care 16, 224 https://doi.org/10.1186/cc11236

Bone RC, Sprung CL, Sibbald WJ (1992): Definitions for sepsis and organ failure. Crit. Care Med. 20, 724-726 https://doi.org/10.1097/00003246-199206000-00002

Bossi F, Peerschke EI, Ghebrehiwet B, Tedesco F (2011): Cross-talk between the complement and the kinin system in vascular permeability. Immunology letters 140, 7-13 https://doi.org/10.1016/j.imlet.2011.06.006

Chereshnev VA, Gusev EYu, Zotova NV (2010): Fundamentalapplied aspects of systemic inflammation in terms of physi- ologic and typical pathological process. Ross. Fiziol. Zh. Im I. M. Sechenova 96, 696-707 (in Russian)

Costello RA, Nehring SM (Updated 2019 May 3): Disseminated Intravascular Coagulation (DIC). In: StatPearls [Internet]. Treasure Island (FL): StatPearls Publishing. Available from: https://www.ncbi.nlm.nih.gov/books/NBK441834/

Dalli J, Colas RA, Quintana C, Barragan-Bradford D, Hurwitz S, Levy BD, Choi AM, Serhan CN, Baron RM (2017): Human sepsis eicosanoid and proresolving lipid mediator temporal profiles: correlations with survival and clinical outcomes. Crit. Care. Med. 45, 58-68 https://doi.org/10.1097/CCM.0000000000002014

Das U (2014): HLA-DR expression, cytokines and bioactive lipids in sepsis. Arch. Med. Sci. 10, 325-335 https://doi.org/10.5114/aoms.2014.42586

De Backer D, Donadello K, Taccone FS, Ospina-Tascon G, Salgado D, Vincent JL (2011): Microcirculatory alterations: potential mechanisms and implications for therapy. Ann. Intensive Care 1, 27 https://doi.org/10.1186/2110-5820-1-27

De Paoli F, Staels B, Chinetti-Gbaguidi G (2014): Macrophage phenotypes and their modulation in atherosclerosis. Circ. J. 78, 1775-1781 https://doi.org/10.1253/circj.CJ-14-0621

Drewry AM, Hotchkiss RS (2015): Sepsis: Revising definitions of sepsis. Nat. Rev. Nephrol. 11, 326-328 https://doi.org/10.1038/nrneph.2015.66

Dunne WM, Jr (2015): Laboratory diagnosis of sepsis? No SIRS, not just yet. J. Clin. Microbiol. 53, 2404-2409 https://doi.org/10.1128/JCM.03681-14

Epstein L, Dantes R, Magill S, Fiore A (2016): Varying estimates of sepsis mortality using death certificates and administrative codes - United States, 1999-2014. Morbidity and Mortality Weekly Report 65, 342-345 https://doi.org/10.15585/mmwr.mm6513a2

Granja C, Povoa P (2015): PIRO and sepsis stratification: reality or a mirage? Rev. Bras. Ter. Intensiva 27, 196-198 https://doi.org/10.5935/0103-507X.20150038

Gusev EY, Chereshnev VA (2013): Systemic inflammation: theoretical and methodological approaches to description of general pathological process model. Part 3. Backgroung for nonsyndromic approach. Patol. Fiziol. Eksp. Ter. 3, 3-14 (in Russian)

Gusev EYu, Yurchenko LN, Chereshnev VA, Zotova NV, Kopalova YuA, inventors; Institute of immunology and physiology UB RAS, assignee (2008): The method for diagnosis and prognosis of Systemic Inflammation with phases and stages verification. Russian Federation Patent RF 2335771, IPC7 G01N 33/53. 2008 Oct 10. Russian

Gusev EY, Zotova NV (2019): Cellular stress and general pathological processes. Curr. Pharm. Des. 25, 251-297 https://doi.org/10.2174/1381612825666190319114641

Higgins JP (2002): Nonlinear systems in medicine. Yale J. Biol. Med. 75, 247-260

Huang W, Tang Y, Li L (2010): HMGB1, a powerful pro-inflammatory cytokines in sepsis. Cytokine 51, 119-126 https://doi.org/10.1016/j.cyto.2010.02.021

Karlsson S, Pettilä V, Tenhunen J, Lund V, Hovilehto S, Ruokonen E (2008): Vascular endothelial growth factor in severe sepsis and septic shock. Anesth. Analg. 106, 1820-1826 
https://doi.org/10.1213/ane.0b013e31816a643f

Kiral E, Dinleyici EC, Bozkurt-Turhan A, Bor O, Akgun Y, Akgun NA (2016): Serum endocan levels in children with febrile neutropenia. Hematol. Rep. 8, 6110 https://doi.org/10.4081/hr.2016.6110

Mahieu T, Libert C (2007): Should we inhibit type I interferons in sepsis? Infect. Immun. 75, 22-29 https://doi.org/10.1128/IAI.00829-06

Maruna P, Gürlich R, Frasko R, Haluzík M (2001): Serum leptin levels in septic men correlate well with C-reactive protein (CRP) and TNF-alpha but not with BMI. Physiol. Res. 50, 589-594

Mat-Nor MB, Md Ralib A, Abdulah NZ, Pickering JW (2016): The diagnostic ability of procalcitonin and interleukin- 6 to differentiate infectious from noninfectious systemic inflammatory response syndrome and to predict mortality. J. Crit. Care 33, 245-251 https://doi.org/10.1016/j.jcrc.2016.01.002

Monneret G, Lepape A, Venet F (2011): A dynamic view of mHLADR expression in management of severe septic patients. Crit. Care 15, 198 https://doi.org/10.1186/cc10452

Panich T, Chancharoenthana W, Somparn P, Issara-Amphorn J, Hirankarn N, Leelahavanichkul A (2017): Urinary exosomal activating transcriptional factor 3 as the early diagnostic biomarker for sepsis-induced acute kidney injury. BMC Nephrol. 18, 10 https://doi.org/10.1186/s12882-016-0415-3

Pierrakos C, Vincent JL (2010): Sepsis biomarkers: a review. Crit. Care 14, R15 https://doi.org/10.1186/cc8872

Pockley AG, Muthana M, Calderwood SK (2008): The dual immunoregulatory roles of stress proteins. Trends Biochem. Sci. 33, $71-79$ https://doi.org/10.1016/j.tibs.2007.10.005

Polat G, Ugan RA, Cadirci E, Halici Z (2017): Sepsis and septic shock: current treatment strategies and new approaches. Eurasian J. Med. 49, 53-58 https://doi.org/10.5152/eurasianjmed.2017.17062

Ricklin D, Reis ES, Lambris JD (2016): Complement in disease: a defence system turning offensive. Nat. Rev. Nephrol. 12, 383-401 https://doi.org/10.1038/nrneph.2016.70

Rioux J (2012): A complex, nonlinear dynamic systems perspective on Ayurveda and Ayurvedic research. J. Altern. Complement. Med. 18, 709-718 https://doi.org/10.1089/acm.2011.0569

Sertaridou E, Papaioannou V, Kolios G, Pneumatikos I (2015): Gut failure in critical care: old school versus new school. Ann. Gastroenterol. 28, 309-322
Sharma D, Kanneganti TD (2016): The cell biology of inflammasomes: Mechanisms of inflammasome activation and regulation. J. Cell Biol. 213, 617-629 https://doi.org/10.1083/jcb.201602089

Silver JH (2011): Nitrite and nitric oxide as potential diagnostic markers in acute vascular diseases. J. Neurol. Neurophysiol. S1

Singer M, Deutschman CS, Seymour CW, Shankar-Hari M, Annane D, Bauer M, Bellomo R, Bernard GR, Chiche JD, Coopersmith CM, et al. (2016): The third international consensus definitions for sepsis and septic shock (Sepsis-3). JAMA 315, 801-810 https://doi.org/10.1001/jama.2016.0287

Takala A, Jousela I, Olkkola KT, Jansson SE, Leirisalo-Repo M, Takkunen O, Repo H (1999): Systemic inflammatory response syndrome without systemic inflammation in acutely ill patients admitted to hospital in a medical emergency. Clin. Sci. (Lond) 96, 287-295 https://doi.org/10.1042/cs0960287

Ter Horst R, Jaeger M, Smeekens SP, Oosting M, Swertz MA, Li Y, Kumar V, Diavatopoulos DA, Jansen AFM, Lemmers H, et al. (2016): Host and environmental factors influencing individual human cytokine responses. Cell 167, 1111-1124 https://doi.org/10.1016/j.cell.2016.10.018

Valenzuela-Sánchez F, Valenzuela-Méndez B, Rodríguez-Gutiérrez JF, Estella-García Á, González-García MÁ (2016): New role of biomarkers: mid-regional pro-adrenomedullin, the biomarker of organ failure. Ann. Transl. Med. 4, 329 https://doi.org/10.21037/atm.2016.08.65

Vincent JL, Beumier M (2013): Diagnostic and prognostic markers in sepsis. Expert Rev. Anti Infect. Ther. 11, 265-275 https://doi.org/10.1586/eri.13.9

Wada H, Matsumoto T, Yamashita Y (2014): Diagnosis and treatment of disseminated intravascular coagulation (DIC) according to four DIC guidelines. J. Int. Care 2, 15 https://doi.org/10.1186/2052-0492-2-15

Wu JF, Ma J, Chen J, Ou-Yang B, Chen MY, Li LF, Liu YJ, Lin AH, Guan XD (2011): Changes of monocyte human leukocyte antigen-DR expression as a reliable predictor of mortality in severe sepsis. Crit. Care 15, R220 https://doi.org/10.1186/cc10457

Zotova NV, Chereshnev VA, Gusev EY (2016): Systemic inflammation: methodological approaches to identification of the common pathological process. PLoS One 11, e0155138 https://doi.org/10.1371/journal.pone.0155138

Zweig MH, Campbell G (1993): Receiver-operating characteristic (ROC) plots: a fundamental evaluation tool in clinical medicine. Clin. Chem. 39, 561-577

Received: May 27, 2019

Final version accepted: September 30, 2019 